\title{
THE FLAGSTAFF MEASURING MACHINE
}

\author{
Gart Westerhout and Dave Monet \\ U.S. Naval Observatory \\ Washington, DC 20392 \\ USA
}

\begin{abstract}
The U.S. Naval Observatory is building a measuring machine which will hold four PSS plates simultaneously and will automatically measure them, in a highly controlled environment, in about 12 hours. The images will be recorded by two camera subsystems with a scale of 0.9 arcseconds/pixel on a $0.33 \times 0.26$ degree CCD frame. The original glass plates of the PSS-II and glass copies of the PSS-I will be measured. This is a joint CALTECH-USNO program.
\end{abstract}

\section{INTRODUCTION}

To our knowledge, no other papers in this Symposium describe the second Palomar Sky Survey (PSS-II). Therefore, we start this paper with a short description. The PSS-II consists of 894 fields on 5-degree centers from declination 0 to 90 degrees, each exposed in three colors:
Blue: III aJ + GG 385
Red: $\quad$ III $\mathrm{aF}+\mathrm{RG} 600$
Near-IR: IV N + RG 9

The plates measure $35 \times 35 \mathrm{~cm}$ or $6.6 \times 6.6$ degrees, and the plate scale is $67.1 \mathrm{arcsec} / \mathrm{mm}$. The blue and red plates go to 23 and 22 magnitude, respectively, which is 2 magnitudes fainter than PSS-I. In addition, the U.S. Naval Observatory has commissioned the taking of a short-exposure ( 2 minute unbaked) blue plate to allow an astrometric tie-in with the fundamental reference system (existing astrometric catalogs). This short plate will be taken within 1 month of the PSS-II blue plate.

The accuracy of relative positions and absolute proper motions measurable on the PSS plates is limited by the $\mathrm{S} / \mathrm{N}$ characteristics and the plate scale to a centroiding accuracy of approximately $1-1.5$ micrometers, corresponding to 0.1 arcseconds. Thus relative positions of $0.1 "$ are expected.

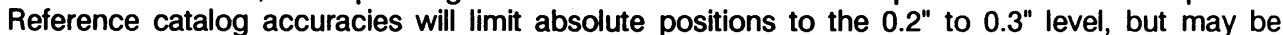
improved with future catalogs. With the PSS 30 -year baseline, proper motions of $0.003^{\prime \prime} /$ year are expected and will be complete from 13 - 19 magnitude outside the Milky Way. It is likely that these will be absolute proper motions, as the number of QSO's per plate at $V=19$ is about 300 .

\section{EXPECTED CATALOG PRODUCTS.}

All processing will be done in real time, but all CCD frames will be retained for future use. The first catalog available will be a Raw Catalog, containing $X$ and $Y$ positions in microns, estimators of object classification, and an uncalibrated density estimate. This will be a plate-by-plate catalog, with no inter-plate correlation nor photometric calibration. Subject to manpower and computer limitations, this Raw Catalog will be available to qualified, active users. We cannot support a general distribution.

The User Catalog will be calibrated both astrometrically and photometrically, using the best existing 
astrometric catalogs and photometry from the STScl guide star catalog and other existing photometric data. The detection lists for each field will be correlated and merged to provide for each object a mean position, mean magnitude, color, proper motion, and object classification. The fields will then be merged into a single catalog. It is also intended to isolate the QSO's to determine the zero point of the proper motion system.

User support documentation to be provided will be the source code for image processing, the source code for list correlation, and the calibration formulae and coefficients that convert the Raw Catalog into the User Catalog. The User Catalog will be widely distributed.

\section{PRECISION MEASURING MICRODENSITOMETER (PMM).}

The only company we have found capable of providing a $30 \times 40$ inch active measuring area (to mount four plates at one time) using granite and air-bearing technology is ANORAD. Metrology in $X$ and $Y$ will be interferometric, with $1 / 16$ wavelength local precision and 0.5 micrometers/meter global. There will be a separate auto-focus on the upper and lower Z-axis stages. The machine will be provided with two measuring CCD cameras and a microdensitometer with a slit-viewing TV camera. It will be housed in a class 100,000 clean room enclosure and kept to within 0.5 degrees C temperature.

Each camera subsystem is a blemish-free VIDEK CCD camera with $1320 \times 1035$ pixels, 8-bit digitization, $10 \mathrm{MHz}$ readout rate, and 6.8 micrometer square pixels. A 2:1 transfer lens will translate this to 13.6 micrometer square pixels on the plate, providing a scale of 0.9 arcseconds/pixel, and a $0.33 \times 0.26$ degree area per CCD frame. The microdensitometer subsystem will be supplied by Perkin-Elmer and will be substantially the same as that used on their PDS machine.

The philosophy behind the computer follows from the condition that we want to mount the four plates, leave them for a while to assume constant temperature, then measure them automatically in about 12 hours. This provides 17 seconds computer time for each frame; the computer speed then dictates how much information can be extracted from a frame in these 17 seconds. The configuration consists of three Silicon-Graphics 4D/240 "advanced workstations" boxes. Each box will have 4 RISC CPUs, 32 MByte Physical Memory, 600 MByte Disk, 1/4 Inch Tape and will use UNIX and FORTRAN-77 with VMS Extensions.

We are coding in FORTRAN, and work in a multi-thread, multi-CPU parallel computational environment. The full-frame operation co-adds four VIDEK images, flattens the field and normalizes, uses a box median filter to get the sky, and uses a star finder and primitive splitter. For each star detection it then does 0 th, 1 st, and 2 nd density moments (total intensity, position and shape), makes gradient and separation measures, and does a non-linear least squares fit.

The PMM will be delivered in December 1990. Software development is in progress, and production is expected to start early in 1991 . The production rate is 1000 plates per year and is expected to catch up with the obsenving in a few years. Total plate material is about 6000 (3 PSS-II, 2 PSS-I, 1 short $\mathrm{J})$, and therefore the total program is expected to take 6 years to accomplish. The final product is a homogeneous catalog of astrometric positions, proper motions, colors, magnitudes, and identifiers for non-stellar objects. 